\title{
An Extension Strategy of Concolic Testing for Intentionally Non-Terminating Programs
}

\author{
Woorak Park ${ }^{1}$, Seungman Han ${ }^{1}$, Cheolgi $\mathrm{Kim}^{2}$, and Euiseong Seo ${ }^{3}$ \\ ${ }^{1}$ Sungkyunwan University, Suwon-si, Gyeonggi-do, Republic of Korea \\ ${ }^{2}$ Software Department, Korea Aerospace University, Goyang-si, Gyeonggi-do, Republic of Korea \\ ${ }^{3}$ College of Software, Sungkyunkwan University, Suwon-si, Gyeonggi-do Republic of Korea
}

\begin{abstract}
Concolic testing (or dynamic symbolic testing) enables simple and efficient software verifications by tracing all possible branches. However, the application of the concolic testing is limited to programs without non-terminating loops although non-terminating loops are essential for embedded control systems or various service programs. This paper proposes an extension to the concolic testing tool that enables verification of intentionally non-terminating programs. The proposed approach records the constraint states of the visited branch and compares whether the newly meeting constraint state has been visited before. The proposed approach was implemented in the KLEE verification tool. The prototype implementation succeeded in the verification of a few programs with intentional non-terminating loops.
\end{abstract}

Keywords: Concolic Test, Dynamic Symbolic Execution, Intentionally Non-terminating Program, KLEE

\section{Introduction}

As the importance of software quality has increased in many fields in recent years, software testing is considered to be a means to improve the software quality[1]. Manual analysis is a traditional method of testing software, but rather automated analysis through software is much better in terms of testing quality and efficiency[2].

The concolic testing is a combination of dynamic analysis and symbolic testing. It obtains information about the reachable paths by adding constraints on every branch during the execution of the program[3]. The utilization of concolic testing tool is being seen as an effective method for software testing, and it is also being actively applied to industry[4].

However, the existing concolic testing methods have various limitations, and the studies are underway to overcome them[5]. One of the limitations of the concolic method is that it is impossible to verify programs containing infinite loops in a reasonable time[6].

There are many intentionally non-terminating programs like various daemon programs. Especially, the control software of embedded systems such as the unmanned aerial vehicle is difficult to verify through concolic testing tools despite its high requirement for verification.

In this paper, we propose a strategy that enables the tests of non-terminating programs by considering the loop bodies as the target programs and connecting their executions. And we applied it to a concolic testing tool, KLEE[7]. 


\section{Background}

\subsection{Related Work}

The fact that the non-terminating programs are hard to be verified through the concolic testing tools originates in the way how the tools work. The verification can be said as completed only when there are no more branches in the execution. There exist tries to modify the search heuristics to achieve high branch coverage for the target which has an infinite loop or a complex control flow.

Rupak Majumdar et al.[6] indicated that the execution time is unreasonable when the random input testing and the depth-first-search(DFS) based concolic testing verify infinitely running and rarely error-causing programs. So they proposed 'Hybrid concolic testing' which is a combination of depth-first-search(DFS) scheme and random input test for better coverage of the particular points.

Taeksu Kim et al.[8] proposed the enhanced DFS as a search heuristic. The enhanced DFS organizes nested loop that explores consequently all neighbor paths instead of recursive exploration. Simultaneously with neighbor path exploration, list of neighbor nodes for next iteration is prepared.

Youngjoo Kim et al.[9] compared various search strategies on KLEE[7]. The compared strategies are DFS scheme, 6 non-uniform random search(NURS) methods offered by KLEE, and breadth-first-search scheme newly implemented by authors.

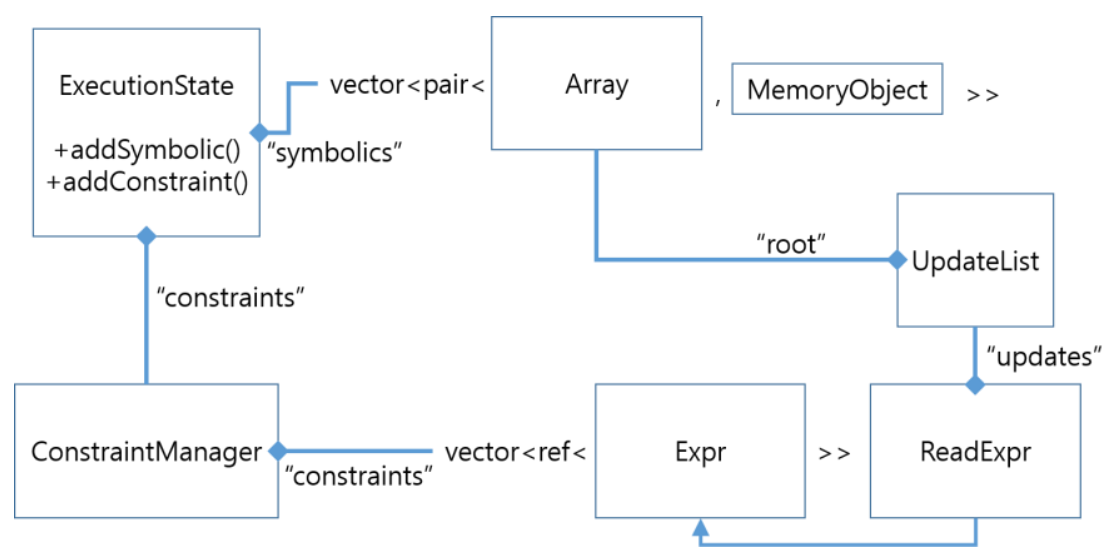

Fig. 1 The management of Symbolics \& Constraints in the KLEE

\subsection{Overview of KLEE Symbolics \& Constraints}

Concolic testing tools need to manage the symbolic variables and the constraints of them. The Fig. 1 shows how to KLEE[7] manage those.

A symbolic variable is represented as a pair of two instances. One is of the class Array which is the logical expression of the variable, and the other is of the class MemoryObject which contains the information of the memory spaces occupied by the variable. And a constraint is represented as an instance of the class Expr which is corresponded to the $\mathrm{C}$ expressions. The singleton instance of the class ExecutionState contains the vectors of the symbolics and the constraints related to the present execution, and it also has the member functions to manage those.

Each constraint is related to some symbolics, so it needs to refer to the symbolics. In order to specify the symbolics in the constraints, the Array instances of the symbolics are included in the instances of ReadExpr which constitute the constraints. 


\section{Concolic Testing for Intentionally Non-terminating Programs}

\subsection{Our Approach}

Intentionally non-terminating programs have loops as their main component. A loop repeats the specific source codes called 'loop body'. If the behavior of the loop body does not change for every iteration, the verification of the loop can be completed by simply verifying its loop body. In the same way, if the behavior of the loop body does change but its cases are finite, the verification of the loop can be completed by verifying its loop body with all of the possible behaviors. Let us call the various possibilities of the behaviors 'states'. The one behavior of the loop body is determined by the bunch of constraints given for one iteration, so the state and the bunch of constraints for that make the one-to-one correspondence.

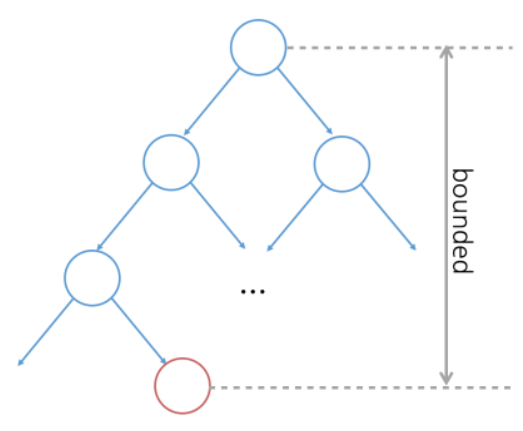

(a)

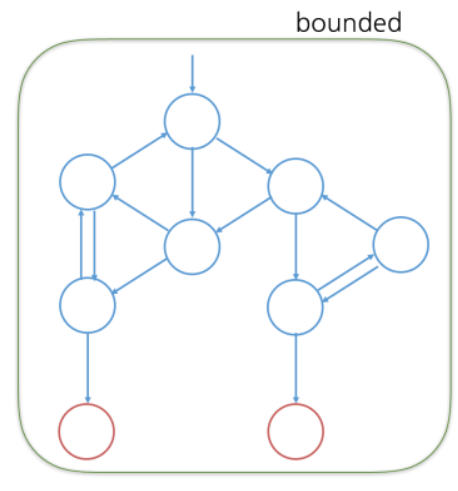

(b)

Fig. 2 The Process of (a) The Existing Concolic Testing and (b) The Proposed Verification Strategy

Fig. 2 presents the process of the existing concolic testing and the concolic testing for a non-terminating program. (a) shows the verification process of a program with finite branch depths by the existing concolic testing, and (b) shows the verification process for a non-terminating program with the bounded states by the proposed strategy.

But it is impossible to guess the exact paths to reach a specific state and whether the state is actually reachable when the only the loop bodies are verified. These problems can be overcome by passing the constraints generated by an iteration to the next iteration and tracking that.

Overall, the verification process of intentionally non-terminating programs is organized as follows.

First, the target program gets the constraints from the former iteration, then add them into its execution state. Second, the target program determines its state with the added constraints. Then the target program replaces its constraints with the ones of all inducible branches from the determined state. Finally, all the branches of the target program pass their constraints to the next iteration and then record the information on them.

\subsection{Implementation}

In order to apply the proposed approach to KLEE, it should be possible to pass the constraints from the current iteration to the next possible iterations and replace the constraints of the current execution state with another's. So we added interfaces in KLEE that can export, import, and clear the constraints of the current execution state. We exploited not only the parts described in section 2 but also the parts that convert constraints to string or vice versa.

And we wrote an additional program in python to connect the loop bodies and manage their states. It collects all the generated constraints from the branches of an iteration, then adds them into its queue. After that, it executes the loop body with the queued constraints except when the constraints have already been applied. Since the applied constraints are put into the Redis[11] database, To check whether the constraints have already been 
applied or not is possible. And the states are covered in the breadth-first-search scheme because the queue is used for the iterations.

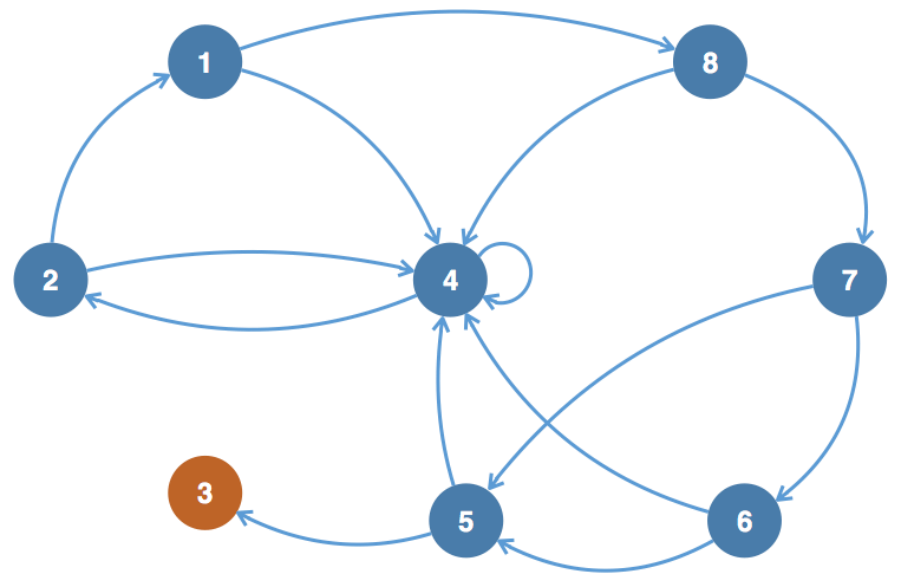

Fig. 3 A Simple Finite State Machine

\section{Evaluation}

\subsection{Possibility of analysis of the intentionally non-terminating program}

Finite state machines containing cycles are the simplest example of the non-terminating programs. Let us consider the finite state machine shown in the Fig. 3. It is a deterministic machine consisting of one variable representing the state and one condition variable determining the direction of the state transition. The state 3 is defined as an error to terminate.

Because all states except the state 3 construct a 'connected graph' of which a state can reach any other state, the error state 3 can be reached from all states eventually.

Since the machine has many cycles, it is difficult to verify it through the existing concolic testing. When we actually tried to verify this machine using existing KLEE, we could observe that KLEE do not search the branches by conditions appropriately but only one direction as shown in Fig. 4. Consequently, It could not present paths to the error state except when it reached accidentally. As with other studies to improve this problem[6][8][9], KLEE also provides several search heuristics including six non-uniform random search(NURS) methods to improve branch searching, and they can be used interleaving[7]. However, every attempt using an unmodified KLEE did not show any improvement within a reasonable time.

In contrast, exactly two transitions are derived from each state according to the condition in the verification through the KLEE-endless and the organized form of the derived states and the transitions is equivalent to the relationship shown in Fig 3. Because the verification through the KLEE-endless reveals what conditions can make the transitions between one to another state, both constraints which make the program errorless and errorprone can be understood. So not only that there exists a path that reaches the error state from all the states, but also the specific paths for that are revealed in the considered machine. For example, the condition comes like ' $\mathrm{T}$ T-F-T-T-T-F-T-T' at each iteration, the fact that the state 4 reaches the error state in the path '4-2-1-4-2-1-8-7-53' precisely can be seen.

As shown above, the existing KLEE fails to satisfy not only the partial correctness, which requires only the correct result in terms of verifying the non-terminating program but also the total correctness which even clears that the verification is over. Whereas the KLEE-endless satisfies the total correctness.

\subsection{Applicability to Physically-Asynchronous, Logically-Synchronized(PALS) System}

MESI protocol[10] is a cache coherence protocol used in symmetric multiprocessing(SMP) system, as the distributed system executed independently at each process. It is physically an asynchronous protocol that has no synchronization method, but it is a logically synchronous protocol that works under the snooped bus operation. 
To check the applicability of the KLEE-endless to the PALS system, we wrote simulated MESI protocol to apply the KLEE-endless. Although the protocol is the distributed, it can be converted into one test target due to its synchronicity.

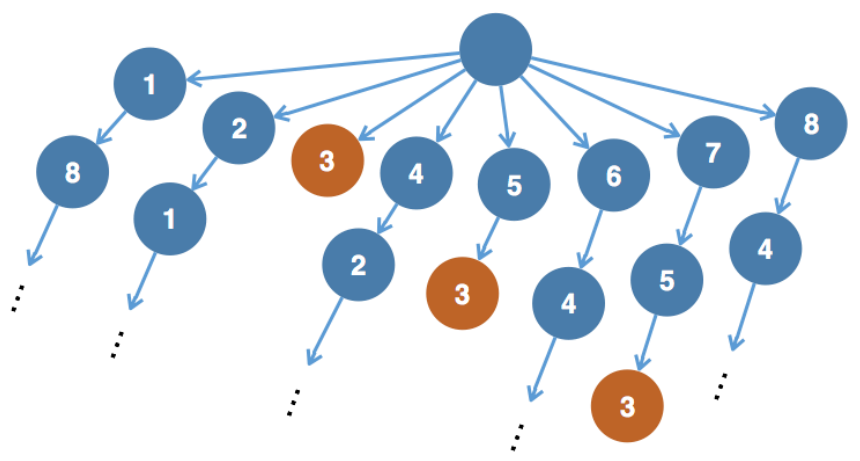

Fig. 4 Inappropriate Searching Behavior of the Existing KLEE

There are the pairs of the states that cannot exist together in the MESI protocol. So if there exists they together, the situation is considered as the violation which means the cache coherence is broken. We analyzed the MESI simulation in terms of the occurrence of violations with 4 simulated processing nodes. When the violation was reached, we skipped to create a state and checked only the violation caused by the bus failure in the normal situation.

No violations occurred when we analyze the normal simulation while 160 states are derived. That means that the MESI protocol is defect-free without any unexpected failures.

Secondly, we assumed there can be a bus failure, which changes the snooped bus operation by a processor randomly at every event. We found 264 possibilities for violations in case of bus failure under normal situation, while about 2300 of states are created. There are 42 kinds of violation, and it can be summarized into 4 kinds in consideration of symmetry. TABLE I presents the kinds of reachable violations, the numbers of normal states causing each kind of the violation, and the examples of the situation causing each violation.

TABLE I: Description on Violation Found by Analysis

\begin{tabular}{|c|c|l|}
\hline $\begin{array}{c}\text { Kind of } \\
\text { violation }\end{array}$ & $\begin{array}{c}\text { No. } \\
\text { cases }\end{array}$ & \multicolumn{1}{|c|}{ Example of the situation causing violation } \\
\hline M E I I & 1 & $\begin{array}{l}\text { 'BusRdX } \rightarrow \text { None' failure occurs at the 'E' state } \\
\text { from 'E I I I' on a 'PrWr' at an 'I' state. }\end{array}$ \\
\hline E S I I & 1 & $\begin{array}{l}\text { 'BusRd } \rightarrow \text { None' failure occurs at the E state } \\
\text { from 'E I I I' on a 'PrRd' at an 'I' state. }\end{array}$ \\
\hline M M I I & 2 & $\begin{array}{l}\text { 'BusRdX } \rightarrow \text { None' failure occurs at the 'M' state } \\
\text { from 'M I I I' on a 'PrWr' at an 'I' state. }\end{array}$ \\
\hline M S I I & 19 & $\begin{array}{l}\text { 'BusRd } \rightarrow \text { None' failure occurs at the 'M' state } \\
\text { from 'M I I I' on a 'PrRd' at an 'I' state. }\end{array}$ \\
\hline
\end{tabular}

The result suggests that MESI protocol with bus failures might not satisfy the cache coherent even though it is defect-free.

Overall, the KLEE-endless can be used to check the existence of errors, and the specific situations causing those even for the PALS systems. The existing KLEE takes an unreasonable time to do that, or it cannot.

\section{Conclusion and Further Research}

The In this paper, we propose an extension strategy to solve the non-verifiability of non-terminating programs, which is one of the big limits of the existing concolic testing, and implemented it based on the 
concolic testing tool, KLEE. As a result, it was possible to verify intentionally non-terminating programs that could not be possible time reasonably using KLEE, and it was possible to derive meaningful verification results. Future studies include application to the programs actually used and the theoretical basis of the strategy.

\section{Acknowledgment}

This work was supported by Institute for Information \& communications Technology Promotion(IITP) grant funded by the Korea government(MSIT) (No. B0101-17-0644, Research on High Performance and Scalable Manycore Operating System)

\section{References}

[1] P. Ammann and J. Offutt, Introduction to Software Testing. Cambridge University Press, 2016, pp. 3-8.

[2] Y. Kim, Y. Kim, Taeksu Kim, Gunwoo Lee, Y. Jang and M. Kim, "Automated unit testing of large industrial embedded software using concolic testing", 28th IEEE/ACM International Conference on Automated Software Engineering (ASE‘13), 2013.

[3] C. Păsăreanu and W. Visser, "A survey of new trends in symbolic execution for software testing and analysis", International Journal on Software Tools for Technology Transfer (STTT'09), vol. 11, no. 4, pp. 339-353, 2009.

[4] M. Kim, Y. Kim and Y. Jang, "Industrial Application of Concolic Testing on Embedded Software: Case Studies", IEEE Fifth International Conference on Software Testing, Verification and Validation(ICST’12), 2012.

[5] X. Qu and B. Robinson, "A Case Study of Concolic Testing Tools and their Limitations", International Symposium on Empirical Software Engineering and Measurement (ESEM'11), 2011.

[6] R. Majumdar and K. Sen, "Hybrid Concolic Testing", 29th International Conference on Software Engineering (ICSE'07), 2007.

[7] C. Cadar, D. Dunbar and D. R. Engler, "KLEE: Unassisted and Automatic Generation of High-Coverage Tests for Complex Systems Programs", 8th USENIX Symposium on Operating Systems Design and Implementation (OSDI'08), pp. 209-224, 2008.

[8] T. Kim, J. Park, I. Kulida and Y. Jang, "Concolic Testing Framework for Industrial Embedded Software", 21st AsiaPacific Software Engineering Conference (APSEC'14), 2014.

[9] Y. Kim, M. Kim, Y. Kim and U. Jung, "Comparison of Search Strategies of KLEE Concolic Testing Tool", Journal of KIISE:Computing Practices and Letters, vol. 18, no. 4, pp. 321-325, 2012.

[10] M. Papamarcos and J. Patel, "A low-overhead coherence solution for multiprocessors with private cache memories", Proceedings of the 11th annual international symposium on Computer architecture (ISCA'84), 1984.

[11] Sanfilippo, S., Redis. https://redis.io, 2009 